\title{
Leitura Compartilhada de Livros e Aprendizagem de Palavras em Crianças Pré-Escolares
}

\author{
Fernanda Pires Garcia \\ Aline Melina Vaz \\ Andréia Schmidt ${ }^{1}$ \\ Faculdade de Filosofia, Ciência e Letras de Ribeirão Preto da Universidade de São Paulo, \\ Ribeirão Preto, SP, Brasil
}

\begin{abstract}
Resumo
A leitura compartilhada de histórias é uma forma eficiente de promover a aprendizagem de vocabulário por crianças, mas os processos básicos envolvidos nessa aprendizagem ainda precisam ser esclarecidos. O objetivo deste estudo foi investigar a influência de dicas sociais na aprendizagem de palavras durante a leitura de livros, e se as novas relações palavras-figuras apresentadas nas histórias sustentariam um desempenho por exclusão posterior, diante de figuras desconhecidas. Duas histórias ilustradas não comerciais, contendo duas pseudopalavras cada, foram lidas com e sem a apresentação de dicas sociais para 12 crianças de 3 anos, em um delineamento de medidas repetidas. Sondas de emparelhamento, exclusão e nomeação avaliaram a aprendizagem dessas pseudopalavras. O desempenho das crianças não diferiu em relação à aprendizagem das pseudopalavras nas duas condições de leitura (com apresentação de dica social e sem dicas), e todas apresentaram desempenho por exclusão nas sondas planejadas. Esses resultados confirmam que a situação de leitura compartilhada promove aprendizagem de novas relações entre palavras e figuras, mas deixam em aberto o papel de dicas sociais nesse processo.
\end{abstract}

Palavras-chave: Leitura compartilhada de livros, aprendizagem de palavras, comportamento verbal, análise do comportamento.

\section{Shared Book Reading and Word Learning in Preschool Children}

\begin{abstract}
Shared story reading is an effective way to promote the learning of vocabulary for children, however, the basic processes involved in this learning have yet to be clarified. The aim of this study was to investigate the influence of social cues on word learning while reading books, and whether the new word-picture relationships presented in the stories sustain subsequent performance by exclusion, given unknown pictures. Two non-commercial illustrated stories, containing two pseudowords each, were read with and without the presentation of social cues for 12 children, 3 years of age, in a repeated measures design. Matching, exclusion and naming probes evaluated the learning of these pseudowords. The performance of the children did not differ in relation to the learning of the pseudowords in both reading conditions
\end{abstract}

Endereço para correspondência: Universidade de São Paulo, Faculdade de Filosofia, Ciências e Letras de Ribeirão Preto, Departamento de Psicologia, Av. Bandeirantes, 3900, Bairro Monte Alegre, Ribeirão Preto, SP, Brasil 14040-901. E-mail: aschmidt@ffclrp.usp.br

A terceira autora é membro do Instituto Nacional de Ciência e Tecnologia sobre Comportamento, Cognição e Ensino (INCT-ECCE), financiado pelo Conselho Nacional de Pesquisa (CNPq, processo 573972/2008-7) e Fundação de Amparo à Pesquisa do Estado de São Paulo (FAPESP, processo \# 08/57705-8). Essa pesquisa foi apoiada por auxílio regular à pesquisa concedido à terceira autora pela FAPESP (processo 2014/22990-5). 
(with presentation of social cues and without cues), and all presented performance by exclusion in the planned probes. These results confirm that the shared reading situation promotes the learning of new relationships between words and pictures, however, do not verify the role of social cues in this process.

Keywords: Shared book reading, word learning, verbal behavior, behavior analysis.

\section{Lectura Compartida de Libros y Aprendizaje de Palavras en Niños en Edad Preescolar}

\section{Resumen}

La lectura compartida de libros es una manera eficaz de promover el aprendizaje de vocabulario de los niños, pero los procesos básicos que intervienen en este aprendizaje todavía necesitan ser aclarado. El objetivo de este estudio fue investigar la influencia de pistas sociales en el aprendizaje de palabras durante la lectura de libros, y se nuevas relaciones nombre-figuras presentado en las historias, mantienen subsecuenteresponder por exclusión, delantenuevas figuras desconocidas. Dos historias ilustradas no comerciales, que contienen dos pseudopalabras cada uno, fueron leídas con y sin la presentación de pistas sociales a 12 niños de 3 años. Pruebas de aprendizaje, exclusión y respuesta verbal evaluaron el aprendizaje de estos pseudopalabras. El desempeño de los niños no difirió en relación al aprendizaje de la pseudopalabra, bajo dos condiciones de la lectura, pero todos mostraron respuesta de exclusión en las pruebas previstas. Estos resultados confirman que la situación lectura compartida promueve el aprendizaje de vocabulario, pero dejan abierta la función de laspistas sociales en este proceso.

Palabras clave: Lectura compartida de libros, aprendizaje de palavras, comportamiento verbal, análisis de la conducta.

O comportamento verbal, de acordo com Skinner (1957), é definido como todo comportamento reforçado pela mediação do ouvinte, que foi ensinado especificamente a se comportar como mediador. Considerando a natureza eminentemente social do comportamento verbal, Skinner defende uma abordagem funcional para o seu estudo: tal comportamento é aprendido na interação do organismo com o seu ambiente social, e esse processo de interação envolve variáveis relacionadas às condições em que uma dada resposta verbal é emitida, as características da própria resposta e as consequências produzidas por este responder (análise das contingências verbais). Nesse sentido, as práticas culturais de uma dada comunidade são cruciais para que os seus membros sejam capazes de aprender repertórios de falantes e de ouvintes, e essas práticas são conduzidas na forma de ensino direto, como quando pais (ou adultos em geral) se engajam ativamente em estabelecer contingências apropriadas para a aprendizagem de repertório verbal, ou de forma incidental, quando a criança é inserida em atividades comuns de seu grupo e exposta a situações que favorecem o desenvolvimento desse repertório.

$\mathrm{Na}$ cultura ocidental, a leitura de livros (ilustrados ou não) para crianças (leitura compartilhada) é uma prática bastante comum e que é referida por muitos autores como uma das atividades mais importantes para a aprendizagem e ampliação do vocabulário em crianças (Elley, 1989; Mol, Bus, de Jong, \& Smeets, 2008), além de fornecer oportunidade para que elas aprendam o significado de novas palavras (Farrant \& Zubrick, 2013). De uma perspectiva comportamental, a ampliação do vocabulário da criança a partir da leitura compartilhada de histórias pode ocorrer em função de uma série de contingências presentes na situação e que favorecem tal aprendizagem. Em algumas delas, a leitura favorece a oportunidade para que a criança relacione diretamente palavras e figuras presentes em livros ilustrados, com ou sem a instrução direta do adulto, de tal forma que ela seja capaz de selecionar a figura correspondente à nova palavra 
ou utilizar a palavra em contexto apropriado (nomear um objeto ou solicitá-lo a um adulto). Isso ocorre, por exemplo, quando o adulto aponta, ou chama a atenção da criança para uma dada figura durante a leitura (pareamento auditivo-visual - Stemmer, 1996; Vichi, Nascimento, \& Souza, 2012), ou pela simples leitura da história enquanto a criança inspeciona as ilustrações do livro, relacionando, sem qualquer instrução direta, as palavras que desconhece na história a elementos da ilustração que também não conhece (desempenho por exclusão - Dixon, 1977).

Em outras ocasiões, a leitura pode favorecer que a criança relacione certas palavras que desconhece a aspectos menos óbvios das ilustrações em função de dicas lexicais presentes nas frases da história (Mintz \& Gleitman, 2002), em um processo mais refinado de controle de estímulos, como, por exemplo, quando uma criança ouve a frase "A maçã estava na cesta magenta": a posição da palavra "magenta" na frase indica que ela tem relação com a palavra "cesta", sendo uma propriedade desta; com isso, a criança pode ficar sob controle de uma propriedade específica da "cesta" - sua cor, o que, neste caso, favoreceria a aprendizagem de um adjetivo. Crianças ainda mais velhas, e com maior experiência com a sintaxe do idioma, seriam capazes não apenas de aprender o significado de palavras novas que aparecem nas histórias, como também a ocasião em que elas são empregadas, e sua relação com outras palavras da língua, apenas por meio de dicas verbais presentes no curso da história. Esses são apenas alguns dos processos de controle de estímulos que podem estar presentes na situação de leitura compartilhada, mas eles têm sido pouco explorados por analistas do comportamento, a despeito da vasta literatura sobre o tema produzida por outras tradições teóricas (e.g., Biemiller \& Boote, 2006; Kucirkova, Messer, \& Sheehy, 2014; Wilkinson \& Houston-Price, 2013).

Especificamente, dois processos têm sido sugeridos por diferentes autores de tradição cognitivista como implicados na aprendizagem de vocabulário em situação de leitura compartilhada por crianças: o mapeamento rápido e as dicas sociopragmáticas. O primeiro deles, referido por Horst (2013) como processo chave na aprendi- zagem de palavras em situação de leitura compartilhada, especialmente em livros ilustrados, é o mapeamento rápido (fast mapping): a criança tende a relacionar as palavras desconhecidas da história aos elementos das ilustrações também desconhecidos, estabelecendo uma apreensão parcial do "significado" da palavra.

$\mathrm{Na}$ Análise do Comportamento, o processo de relacionar estímulos indefinidos, em contraste com estímulos conhecidos, é estudado como "responder por exclusão" (Dixon, 1977). Ao ouvir uma história, a criança pode entrar em contato com uma palavra desconhecida para ela (e.g., "hipopótamo"). Ao olhar a ilustração da página, a criança vê a figura de um elefante, de um leão (ambos conhecidos) e um hipopótamo (desconhecido). Sem qualquer instrução direta, a criança tende a relacionar a palavra desconhecida à figura não familiar, excluindo as demais figuras conhecidas da ilustração como potenciais "referentes" para a palavra nova.

Esse desempenho emergente (não ensinado), é definido como a seleção imediata de um estímulo de comparação indefinido (figura ou objeto desconhecido, que não possui relação com qualquer estímulo específico do ambiente) diante de um estímulo modelo também indefinido (por exemplo, uma palavra falada desconhecida), sem uma história anterior que torne o estímulo de comparação discriminativo para a resposta de seleção (Oshiro, de Souza, \& Costa, 2006). Esse processo tem sido identificado por muitos autores como subjacente à aprendizagem de vocabulário em diferentes situações (e.g., Carey \& Bartlett, 1978; Halberda, 2006; Heibeck \& Markman, 1987; McIlvane \& Stoddard, 1981; Wilkinson, Dube, \& McIlvane, 1998). Evidências de ocorrência do responder por exclusão em situação de leitura compartilhada de livros foram encontradas por Horst, Parsons e Bryan (2011), em pesquisa realizada com crianças de 3 anos, que demonstrou que crianças são capazes de relacionar palavras novas a elementos desconhecidos nas ilustrações de um livro sem o ensino direto dessas relações

Outro processo citado como potencialmente importante como auxiliar na aprendizagem de novas palavras na situação de leitura comparti- 
lhada de histórias são as chamadas dicas sociopragmáticas. Segundo Farrant e Zubrick (2013), a leitura de livros pelo adulto em ambiente natural envolve uma situação estruturada em que as dicas sociais fornecidas (gestos de apontar para as figuras das histórias, a nomeação de aspectos específicos da ilustração e o estabelecimento de atenção compartilhada, por exemplo) promovem uma oportunidade para a criança aprender a mapear referentes correspondentes a palavras desconhecidas presentes na história. Exemplos de dicas sociais podem ser a expressão facial, gestos, direção do olhar, apontar, dar ênfase, entre outras possibilidades.

Várias pesquisas sobre aquisição de vocabulário mostram evidências da importância de pistas sociais para a aprendizagem de relações entre palavras e seus referentes. Um estudo longitudinal realizado por Thoermer e Sodian (2001) com crianças entre 10 e 12 meses de idade, por exemplo, mostrou que os bebês acompanhavam os gestos comunicativos dos adultos, ainda que, inicialmente, sem compreender a relação intencional entre o gesto e o referente, independentemente da situação comunicativa. Um estudo realizado por Rowe (2000) indicou que mães que conversam com frequência com seus bebês apresentam, também com alta frequência, o gesto de apontar. Booth, McGregor e Rohlfing (2008), em estudo com 80 crianças entre 28 a 31 meses, verificaram que a sobreposição de dicas sociais (olhar e apontar, por exemplo, ou olhar e manipular objetos) na situação de nomeação de objetos desconhecidos das crianças tem efeitos mais positivos sobre a atenção e o desempenho das crianças em tarefas de aprendizagem, do que as dicas sociais isoladas.

Além disso, há evidências empíricas de que bebês que apresentam atenção compartilhada, dirigindo sua atenção para o mesmo foco (ação ou objeto) da direção do olhar do adulto, durante um episódio de interação verbal, estabelecem melhor a relação entre palavras e seus referentes (Carpenter, Nagell, \& Tomasello, 1998; Yu \& Smith, 2012). Um estudo de Yu e Ballard (2007), no qual vídeos de interações entre mães e seus bebês são analisados, mostra que os bebês aprendem o significado de palavras em função da frequência de sua ocorrência diante de seus referentes, assim como em função de dicas sociais fornecidas por adultos. Nesse estudo, as autoras verificaram que as mães tendem a sincronizar sua nomeação com a direção de seu olhar e com gestos (como apontar em direção aos objetos nomeados), enquanto os bebês estão olhando para esses objetos ou manipulando-os. Todos esses dados sugerem que há um efeito facilitador de pistas gestuais no aprendizado de palavras.

Não foram encontradas, no entanto, pesquisas que investigassem especificamente o efeito da dica de apontar para ilustrações de livros sobre a aprendizagem de novas palavras na situação de leitura compartilhada. De modo geral, as dicas investigadas com maior frequência nas pesquisas sobre leitura compartilhada são as dicas verbais: fazer perguntas de diferentes tipos (Blewitt, Rump, Shealy, \& Cook, 2009), explicar o significado de palavras novas (Biemiller $\&$ Boote, 2006), nomear especificamente os elementos indefinidos da história (Justice, 2002), fazer comentários sobre as palavras novas (Ard \& Beverly, 2004), dar prompts e feedbacks para comentários do participante (Flores, Pires, \& Souza, 2014) ou apresentar as palavras novas em múltiplos contextos (Wilkinson \& Houston-Price, 2013). Dicas verbais, no entanto, parecem ser mais úteis para falantes mais experientes, capazes de ficar sob controle de aspectos semânticos e lexicais das frases. Crianças mais jovens, porém, parecem se beneficiar fortemente das ilustrações presentes nos livros infantis (Evans \& Saint-Aubin, 2013; Ganea, Allen, Butler, Carey, $\&$ DeLoache, 2009) e dicas gestuais que atuem sobre esse componente das histórias podem ser relevantes na aprendizagem dessas crianças.

Além disso, as medidas de aprendizagem utilizadas pelos estudos sobre aprendizagem a partir da leitura compartilhada de histórias são variáveis, envolvendo a mensuração do número de palavras aprendidas após a situação de leitura (medida em testes de seleção de figuras a partir de uma palavra modelo ditada - e.g., "Mostre para mim onde está o 'blicket'"), ou medidas gerais de vocabulário, aferidas por testes como o Peabody Pictures Vocabulary Test - PPVT (Dunn \& Dunn, 1997), ou, ainda, em provas que avaliam 
se a criança é capaz de descrever o significado de certas palavras. É preciso considerar, no entanto, que a aprendizagem de palavras é um processo que envolve diferentes repertórios, que vão desde a discriminação de que determinada palavra já foi ouvida (ou que o objeto/figura/propriedade a que a palavra se refere não é completamente desconhecido), até ser capaz de empregar a palavra aprendida em contexto apropriado (e.g., nomeação ou o emprego da palavra em uma situação de solicitação). É preciso considerar, porém, a importância de se avaliar a aprendizagem das palavras a que efetivamente a criança foi exposta na situação de leitura, mas em diferentes contextos. Não parece lógico esperar que a situação de leitura compartilhada produza um aumento geral no vocabulário da criança, mas é pertinente esperar que a aprendizagem de determinados vocábulos presentes na história lida possa se refletir em diferentes repertórios da criança.

Mantendo controladas outras variáveis, como as ilustrações do livro e o número de repetições das palavras desconhecidas, pretendeu-se investigar o efeito que uma dica social específica - o gesto de apontar para ilustrações de um livro - pode ter na aprendizagem de novas palavras por meio da leitura de histórias. O objetivo dessa pesquisa foi verificar se crianças na faixa etária de três anos podem aprender mais facilmente relações nome-figura a partir da leitura de histórias ilustradas, lidas para elas com a apresentação de dicas sociais fornecidas por um adulto, em comparação com a leitura sem tais dicas. As medidas de aprendizagem empregadas foram específicas em relação às palavras a que as crianças foram efetivamente expostas durante a leitura: o reconhecimento (ou não) de um contato prévio com a palavra indefinida (sonda de exclusão), a seleção do objeto correspondente à pseudopalavra ditada (sonda de emparelhamento ao modelo) e a nomeação da figura desconhecida que apareceu na história (sonda de nomeação).

\section{Método}

\section{Participantes}

Participaram deste estudo 12 crianças de três anos de idade, que frequentavam uma cre- che em uma cidade do interior de São Paulo, sem indicativos de atraso no desenvolvimento, de acordo com o relato das professoras. Os pais foram informados sobre a pesquisa e autorizaram a participação dos filhos por meio de um termo de consentimento livre e esclarecido.

\section{Materiais}

Foram inventadas seis pseudopalavras (trissílabas) para o presente estudo: fulito, patuce, vicopa, xineque, niveco e pafile. Além disso, foram confeccionados seis objetos de formatos indefinidos, feitos com sucata e papeis coloridos. No presente estudo, tais objetos foram nomeados como "objetos indefinidos" (por não se relacionarem previamente a qualquer palavras da língua), e foram atribuídos, por sorteio, como referentes das pseudopalavras. Quatro dessas pseudopalavras e seus respectivos objetos indefinidos referentes apreciam, em pares, em dois livros de história. Os outros dois objetos indefinidos e pseudopalavras foram utilizados nas Sondas de Exclusão.

Os dois livros com histórias infantis (Livro 1 e Livro 2), ambos com 11 páginas, foram construídos com conteúdo adequado à idade das crianças (descrevendo situações cotidianas como, por exemplo, "Estava fazendo um dia lindo, estava calor, todos estavam contentes e tinham o dia todo para aproveitar. Amanda estava com medo de estragar seu fulito, então resolveu guardá-lo para poder brincar no balanço sem medo de quebrar seu brinquedo"). As páginas de ambos os livros eram compostas de fotos ilustrativas e um pequeno texto (de três a seis linhas, fonte Arial, 28), que era apresentado logo abaixo da figura, seguindo o formato de diversos livros infantis comerciais (ver Figura 1). Em cada livro, um par de pseudopalavras era apresentado. As peusopalavras "Fulito" e "Xineque" apareciam no Livro 1, e "Vicopa" e "Patuci" no Livro 2. Cada pseudopalavra era apresentada três vezes ao longo da história, acompanhada da ilustração de seu respectivo objeto indefinido. Em nenhuma das ilustrações dos livros, as imagens dos dois objetos indefinidos apareciam simultaneamente. 


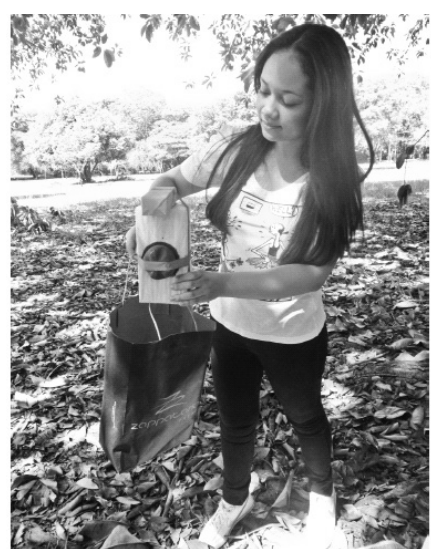

Amanda estava com medo de estragar seu fulito, então resolveu guardá-lo para poder brincar no balanço sem medo de quebrar seu brinquedo.

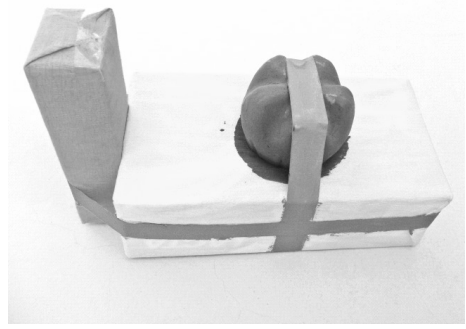

FULITO

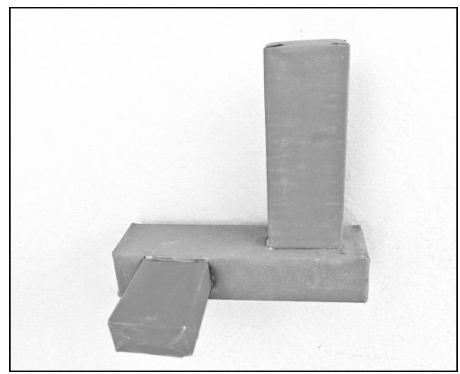

VICOPA

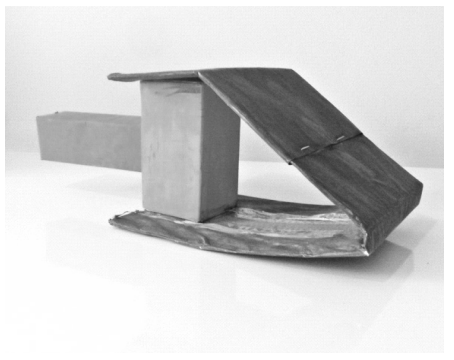

XINEQUE

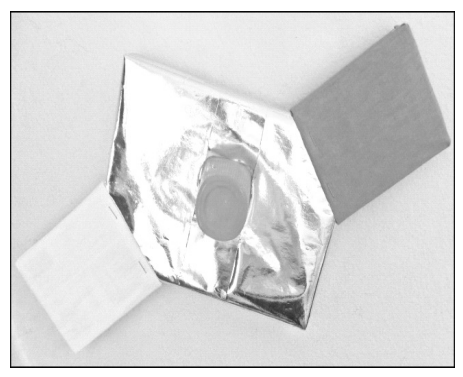

PATUCI

Figura 1. Exemplo de página do Livro 1 (esquerda) e figuras dos objetos indefinidos que apareciam nos Livros 1 e 2 (direita).

Para a verificação da aprendizagem das pseudopalavras foi confeccionado também um Livro de Testes para cada livro de histórias, contendo 16 páginas. Em cada uma das primeiras oito páginas desse livro era apresentado um conjunto de quatro figuras (uma em cada quadrante) e, em cada uma, a pesquisadora pedia que a criança apontasse para uma das figuras conforme um nome ditado (e.g., "Onde está o carro?", ou "Aponte para mim onde está o Patuci"). Nas oito últimas páginas do livro, uma única figura era apresentada por página. Na sequência, serão descritas as tarefas das crianças em cada página.

As quatro primeiras páginas do Livro de Testes continham apenas figuras de objetos conhecidos (e.g., cachorro, carrinho de brinquedo), a fim de levar a criança a compreender a tarefa de discriminação auditivo-visual (selecionar uma figura diante de um nome ditado pela pesquisadora. Nas duas páginas seguintes, eram apresentadas as duas figuras dos objetos indefinidos presentes no livro e duas figuras de brinquedos conhecidos. Nessas páginas eram aplicadas as sondas de emparelhamento ao modelo, em que a criança deveria selecionar a figura correspondente à pseudopalavra ditada (uma tentativa para cada palavra). As duas páginas seguintes eram empregadas para a aplicação das duas Sondas de Exclusão. Nessas duas páginas eram apresentadas as figuras dos objetos indefinidos presentes na história, a figura de um objeto indefinido completamente novo e a figura de um brinquedo conhecido.

Finalmente, nas últimas oito páginas do livro de testes eram apresentadas individualmente as duas figuras dos dois objetos indefinidos presentes na história, entre seis figuras de objetos conhecidos pelas crianças. A cada figura e pesquisadora perguntava qual o nome do objeto apresentado na figura (Sondas de Nomeação).

\section{Procedimentos}

Após um período de ambientação da pesquisadora com as crianças participantes em suas classes, cada criança era convidada, individualmente, para ir para uma sala para ouvir uma his- 
tória. A leitura poderia ser conduzida em duas condições distintas.

Na Condição "Sem Dica" (SD), a pesquisadora lia o livro de forma sequencial, sem interrupções e sem fazer qualquer gesto em direção às figuras. Ao terminar a primeira leitura, a pesquisadora olhava em direção à criança e dizia: "Vou ler mais uma vez", e iniciava a segunda leitura. $\mathrm{O}$ mesmo acontecia para a terceira leitura do livro. Na Condição "Com Dica" (CD) também foram feitas três leituras do livro e, durante cada leitura do livro (mas sem interrompê-la), a pesquisadora colocava o dedo (apontava) para a figura correspondente à pseudopalavra, quando esta era lida na história (“dica social"). Foi tomado cuidado para que não fossem feitas pausas ou dada ênfase às pseudopalavras quando da apresentação das dicas. Como cada uma das duas figuras indefinidas aparecia três vezes na história, em cada uma das leituras a dica foi fornecida em uma página diferente do livro. No total, na Condição $\mathrm{CD}$ a pesquisadora apontou três vezes para cada uma das figuras indefinidas (uma dica para cada pseudopalavra, a cada leitura). Todos os participantes receberam as mesmas dicas.

Todas as crianças passaram pelas duas condições de leitura, com uma semana de intervalo entre as condições (delineamento de medidas repetidas - Cozby, 2003). Para metade das crianças, a Condição SD foi conduzida com o Livro 1 e a Condição CD com o Livro 2. Para a outra metade, foi feito o inverso. A ordem de apresentação das condições também foi contrabalanceada em relação aos participantes. Nas duas condições, logo após as três leituras do livro foram realizadas as sondas de emparelhamento, de exclusão e nomeação. Nas Sondas de Emparelhamento era solicitado à criança que apontasse para uma das quatro figuras presentes na página após seus nomes serem ditados pela pesquisadora (e.g., "Onde está a bola?”, ou "Onde está o Xineque?”). Nas Sondas de Exclusão, duas pseudopalvras completamente novas, que não apareciam nas histórias, foram ditadas, e a criança deveria apontar para a figura de um objeto indefinido completamente novo presente apenas no Livro de Testes. Nas Sondas de Nomeação a criança deveria responder qual o nome da figu- ra apresentada ("Qual é o nome dessa figura?"), tanto para as figuras de objetos definidos como para as de objetos indefinidos.

O conjunto de sondas, portanto, verificava se: (a) a criança aprendeu a relação pseudopalavra-figura indefinida a partir da apresentação destas relações durante a leitura da história; (b) se ela era capaz de discriminar figuras de objetos indefinidos presentes na história (e seus respectivos nomes) de figuras indefinidas e pseudopalavras completamente novas, em sondas de responder por exclusão, selecionando a figura indefinida nova diante de uma nova pseudopalavras; (c) se a criança seria capaz de nomear as figuras indefinidas apresentadas no livro de histórias.

O desempenho das crianças na apresentação das sondas foi filmado. Durante as sondas, a pesquisadora anotava em folha de registro a resposta dada pela criança e as anotações foram comparadas com as filmagens. Para análise dos dados, foram computados acertos e erros dos participantes nas sondas de emparelhamento, de exclusão e de nomeação, nas duas condições. A aprendizagem das pseudopalavras, avaliada pela frequência absoluta de acertos nas sondas de emparelhamento, foi comparada nas condições CD e SD por meio do teste não paramétrico de Wilcoxon. As porcentagens de acertos das pseudopalavras, em suas respectivas condições, foram comparadas por meio do teste não paramétrico de Friedman, seguido de comparações múltiplas pareadas pelo teste de Nemenyi. O nível de significância utilizado foi de 0,05 , sendo tal análise realizada no programa XLStat, versão 2014. A análise de acertos e erros das crianças nas sondas de exclusão e nomeação foi feita de forma descritiva.

Esta pesquisa teve a aprovação do Comitê de Ética em Pesquisa da Faculdade de Filosofia, Ciências e Letras de Ribeirão Preto da Universidade de São Paulo - USP (Certificado de Apresentação para Apreciação Ética [CAAE] 15832813.0.0000.5407).

\section{Resultados}

Todos os participantes demonstraram compreensão da tarefa de discriminação auditivo- 
-visual, selecionando corretamente as figuras de objetos conhecidos diante de seus nomes nas quatro primeiras tentativas com o livro de testes. Nas duas condições, comparando-se o desempe- nho apenas nas Sondas de Seleção, pôde-se observar a ocorrência de aprendizagem parcial das relações nome-figura, conforme apresentado na Tabela 1.

Tabela 1

Acertos ( $\sqrt{ }$ ) e Erros (-) dos Participantes Individuais nas Sondas de Emparelhamento, por Pseudopalavra, nas Duas Condições de Leitura (CD - Com Dica; SD - Sem Dica). Cada Pseudopalavra foi Testada Uma Vez

\begin{tabular}{ccccc}
\hline & \multicolumn{2}{c}{ Condição CD } & \multicolumn{2}{c}{ Condição SD } \\
\cline { 2 - 5 } Participante & Palavra 1 & Palavra 2 & Palavra 1 & Palavra 2 \\
\cline { 2 - 5 } P1 & - & - & - & - \\
P2 & - & $\sqrt{ }$ & - & $\sqrt{ }$ \\
P3 & $\sqrt{ }$ & $\sqrt{ }$ & - & - \\
P4 & $\sqrt{ }$ & $\sqrt{ }$ & $\sqrt{ }$ \\
P5 & $\sqrt{ }$ & - & $\sqrt{ }$ & $\sqrt{ }$ \\
P6 & - & - & $\sqrt{ }$ & - \\
P7 & $\sqrt{ }$ & $\sqrt{ }$ & $\sqrt{ }$ \\
P8 & - & - & $\sqrt{ }$ & $\sqrt{ }$ \\
P9 & $\sqrt{ }$ & $\sqrt{ }$ & $\sqrt{ }$ & - \\
P10 & $\sqrt{ }$ & $\sqrt{ }$ & - & - \\
P11 & $\sqrt{ }$ & - & - & $\sqrt{ }$ \\
P12 & $\sqrt{ }$ & $\sqrt{ }$ & & \\
\hline
\end{tabular}

$\mathrm{Na}$ Condição SD, tomando-se os dados do grupo, foram computados 14 acertos de 24 possibilidades; seis participantes apresentaram respostas corretas nas duas sondas, mas quatro não apresentaram aprendizagem de nenhuma das duas palavras. A média de aprendizagem foi de 1,17 palavras. Na Condição CD foram computados 15 acertos e, novamente, seis participantes acertaram todas as sondas; três crianças, no entanto, não demonstraram aprendizagem de nenhuma das duas palavras. Nesta condição, a média de acertos dos participantes foi de 1,25 palavras. Ao realizar a comparação pareada, não foi verificada diferença estatística significativa entre as duas condições $(W=3,0 ; p=0,68)$. Quatro crianças demonstraram aprendizagem das duas palavras nos dois livros, e apenas uma criança não demonstrou aprendizagem de nenhuma das palavras. De modo geral, os resultados alcança- dos nas sondas de seleção sugerem que não houve diferença entre as duas condições propostas neste estudo. $\mathrm{Na}$ avaliação pareada dos acertos das quatro palavras nas duas condições em análise, observou-se a ocorrência do mesmo padrão de aprendizagem $(\mathrm{Q}=0,36 ; p>0,05)$, indicando que nenhuma das quatro pseudopalavras empregadas nas histórias tinha características específicas que pudessem facilitar ou dificultar especificamente sua aprendizagem.

Nas Sondas de Exclusão, tinha-se o objetivo de verificar se as crianças seriam capazes de discriminar as pseudopalavras apresentadas na história de outras pseudopalavras, e se a dica social favoreceria essa discriminação. Os resultados indicam que todas as crianças foram capazes de fazer essa discriminação, independente da condição de leitura: a dica de apontar não exerceu nenhuma influência sobre essa medida. Na Con- 
dição $\mathrm{CD}, 11$ de 12 participantes acertaram as duas sondas de exclusão (apenas P10 selecionou o estímulo relacionado à palavra "Xineque" na história); na Condição SD, todos os 12 participantes responderam por exclusão.

Apenas três participantes apresentaram respostas de nomeação para as figuras indefinidas. Na Condição CD, P4 (um participante que demonstrou aprendizagem das duas palavras nas Sondas de Seleção) conseguiu nomear os dois objetos indefinidos, e P11 conseguiu nomear um dos objetos indefinidos de forma correta. $\mathrm{Na}$ Condição SD, apenas $\mathrm{P} 2$ conseguiu nomear corretamente um dos objetos indefinidos.

\section{Discussão}

O presente estudo teve o objetivo de investigar se crianças na faixa etária de três anos aprenderiam mais facilmente relações nome-figura a partir de histórias ilustradas lidas de forma dirigida (com dicas sociais), em comparação com a leitura de histórias sem a presença dessas dicas. Na presente pesquisa, metade dos participantes demonstrou aprendizagem das duas palavras presentes na história lida, independente da condição experimental (com ou sem a dica de apontar para as figuras), o que indica que a variável independente manipulada, a dica social, não facilitou nem dificultou a aprendizagem das novas relações palavra-figura. Além disso, todas as crianças, independente da condição de leitura, responderam por exclusão nas sondas planejadas para esse fim, indicando que a leitura de histórias que contenham palavras e figuras desconhecidas produzem, no mínimo, um nível inicial de aprendizagem: no caso aqui relatado, todas as crianças foram capazes de discriminar as pseudopalavras e as figuras de objetos indefinidos presentes na história, de outras pseudopalavras de figuras de objetos indefinidos, totalmente novos.

De modo geral, os resultados aqui obtidos indicam que: (a) crianças na faixa etária de 3 anos podem aprender palavras completamente desconhecidas a partir de um pequeno número de exposições à leitura de um livro ilustrado; (b) a despeito de dados da literatura sobre o papel de dicas gestuais na aprendizagem de vocabulário, não foram encontradas evidências de um papel facilitador da dica de apontar sobre essa aprendizagem na situação investigada; (c) o que se convenciona chamar de "aprendizagem de vocabulário" a partir da situação de leitura compartilhada parece ser um conjunto de diferentes repertórios, que vão desde os mais simples (discriminar a relação palavra-figura desconhecidas presentes no livro das demais), até os mais complexos, como nomear as figuras de objetos indefinidos. Esses pontos serão discutidos na sequência.

O primeiro ponto a ser destacado é que a preparação do material empregado na pesquisa buscou isolar o efeito da leitura da experiência prévia das crianças. Algumas pesquisas sobre o efeito da leitura compartilhada na aprendizagem de novas palavras utilizam palavras correntes na língua dos participantes, ou livros disponíveis no mercado editorial (Robbins \& Ehri, 1994; Wilkinson \& Houston-Price, 2013; Zucker, Cabell, Justice, Pentimonti, \& Kaderavek, 2013). Esse tipo de material tem a vantagem de não requerer a criação de enredos específicos para a situação de pesquisa e contam com recursos visuais atraentes para as crianças, o que pode favorecer seu engajamento, além de aproximarem mais a pesquisa de uma situação natural. Por outro lado, o uso de livros comerciais em pesquisas básicas, cujo objetivo seja entender os processos envolvidos na aprendizagem de vocabulário, tem a desvantagem de não controlar de forma segura o contato prévio (ou adicional, ao longo da pesquisa) dos participantes com as palavras ensinadas. Na presente pesquisa, assim como na de Horst et al. (2011), os livros, as pseudopalavras e as figuras empregadas foram criadas pelas pesquisadoras, o que garantiu que a eventual aprendizagem das palavras ocorreu em função do procedimento de leitura conduzido, e não de um contato anterior das crianças com as palavras utilizadas ou com as figuras apresentadas.

O conjunto de dados aqui apresentados é compatível com os dados de outras pesquisas que utilizam a leitura de livros como forma de ensino de palavras para crianças pré-escolares e em idade escolar (e.g., Flores et al., 2014; Horst et al., 2011; Robbins \& Ehri, 1994; Wilkinson \& Houston-Price, 2013). Considerando que quatro 
crianças demonstraram aprendizagem de todas as palavras novas apresentadas nos dois livros nas sondas de emparelhamento, e que outras quatro crianças demonstraram esse desempenho em relação a todas as palavras de pelo menos um livro (ainda que a variável independente - apontar - não tenha se mostrado crítica no presente estudo), parece haver indícios fortes de que, a atividade de ler para crianças bastante jovens é uma situação propícia para a aprendizagem de vocabulário novo, dadas certas condições.

Ainda não se tem clareza sobre quais seriam as condições necessárias e suficientes para que a aprendizagem via leitura de histórias ocorra, mas, como afirma Horst (2013), certamente os mesmos processos envolvidos na aprendizagem de palavras novas em condições naturais de interação entre a criança e seu contexto social estão implicados na aprendizagem de vocabulário na situação de leitura de histórias ilustradas. Quando uma palavra desconhecida é apresentada em uma situação de leitura (da mesma forma como quando é apresentada em uma situação de interação verbal com um adulto ou um par), a criança precisa ficar sob controle da sequência de sons que compõem a nova palavra, assim como sob controle de dicas que estabeleçam qual o aspecto do mundo a que a palavra se refere. Apresentações repetidas da mesma palavra em diferentes contextos, seja com o pareamento direto com seu referente (como quando a palavra é apresentada na presença de apenas uma figura), seja contrastando elementos conhecidos com a figura desconhecida a que a palavra se refere, ou simplesmente a partir de dicas verbais (e.g., posição da palavras na frase, dicas lexicais específicas) promovem o contato repetido da criança com as novas relações, ampliando as possibilidades de estabelecimento do "significado" da palavra.

No caso específico da leitura de livros para crianças pré-escolares, as ilustrações desempenham um papel muito importante no estabelecimento dessas relações de controle de estímulos. Várias pesquisas indicam que crianças pequenas gastam mais tempo olhando em direção às ilustrações de um livro do que em direção ao texto (e.g., Evans \& Saint-Aubin, 2005; Justice, Skibbe, Canning, \& Lankford, 2005). Além dis- so, Evans e Saint-Aubin (2013), em um estudo sobre movimento do olhar, mostraram que as crianças sincronizam a direção do seu olhar na ilustração com as palavras que são lidas na história, inspecionando por mais tempo os elementos desconhecidos do que os conhecidos. Nesse caso específico, o desempenho por exclusão parece importante.

Dadas as condições apropriadas, como, por exemplo, uma quantidade massiva de palavras conhecidas na história e uma proporção pequena de palavras desconhecidas (Brown, Waring, \& Donkaewbua, 2008), e ilustrações que apresentem os elementos desconhecidos (Evans \& Saint-Aubin, 2013) estabelece-se o contraste necessário entre estímulos "conhecidos" (palavras familiares faladas pelo leitor e as figuras das ilustrações) e estímulos desconhecidos (palavras não familiares que ocorrem na história e aspectos desconhecidos das ilustrações). Sem esse contraste, ou seja, sem uma linha de base estabelecida entre a maior parte das palavras presentes na história e os elementos das ilustrações, dificilmente a criança conseguiria identificar, por exclusão, os estímulos desconhecidos da história (Wilkinson, de Souza, \& McIlvane, 2000). No presente estudo, a evidência de que as crianças fizeram isso ao longo das leituras repetidas é que, nas sondas de emparelhamento ao modelo, pelo menos metade das crianças relacionaram as palavras desconhecidas às figuras dos objetos desconhecidos.

Esperava-se, nesta pesquisa, que a dica de apontar do adulto proporcionasse melhores condições para que a relação entre as pseudopalavras e os objetos indefinidos presentes nas ilustrações se estabelecesse, melhorando, desta forma, o controle exercido pela figura sobre a atenção da criança. No entanto, a despeito do importante papel desempenhado pelo contexto das ilustrações sobre a aprendizagem, não foi possível esclarecer a contribuição da presença ou ausência da dica de apontar para este tipo de tarefa. Vários estudos buscam entender de que maneira dicas gestuais, como o apontar e o olhar, apoiam a aprendizagem precoce de palavras em diferentes situações (e.g., Thoermer \& Sodian, 2001; Tomasello, Carpenter, \& Liszkowski, 2007), e há dados que indicam que, em situações 
de interação verbal entre adultos e crianças, as dicas gestuais de adultos são importantes para a aprendizagem de novas palavras por crianças (Booth et al., 2008). O presente estudo, porém, não identificou diferença significativa na aprendizagem das relações pseudopalavras-figuras quando os participantes foram submetidos à condição $\mathrm{CD}$, comparados à condição SD. Pode-se supor que o número de palavras indefinidas apresentadas em conjunto na história (duas) e o número de exposições de cada uma delas (nove) não tenham sido suficientes para que a presença da dica tenha tido, de fato, algum efeito facilitador na aprendizagem. Pesquisas futuras podem investigar se o aumento do número de palavras desconhecidas na história ou a variação das dicas gestuais apresentadas para a criança durante a leitura exercem um efeito diferencial sobre a aprendizagem estudada.

Finalmente, é preciso ressaltar que o conjunto de sondas realizadas no presente estudo indica que, de fato, a aprendizagem de palavras novas em quaisquer situações (inclusive durante a leitura compartilhada de histórias) envolve vários repertórios (Greer \& Du, 2015; Horst, 2013; Skinner, 1957), de diferentes naturezas e funcionalmente distintos, e que compõem, em conjunto, o que se costuma chamar de vocabulário expressivo e receptivo. Uma análise ampla desses diferentes repertórios pode ser de grande utilidade para se entender de que maneira essa aprendizagem se constrói ao longo do contato da criança com sua comunidade verbal e indicar caminhos sobre as variáveis críticas para cada um nas diferentes situações de aprendizagem.

Todas as crianças que participaram desta pesquisa apresentaram algum tipo de aprendizagem: P4, por exemplo, foi capaz discriminar uma palavra completamente nova das palavras desconhecidas ouvidas durante a história (sonda de exclusão), relacionou corretamente todas as pseudopalavras às suas figuras correspondentes nas sondas de emparelhamento e nomeou duas das figuras; P11, por sua vez, conseguiu nomear uma única figura desconhecida, a mesma que relacionou corretamente ao seu nome na sonda de emparelhamento, mas não teve desempenho semelhante em relação às demais relações nome- -figura; e P1 não relacionou corretamente nenhuma das peseudopalavras às suas figuras nas quatro sondas de emparelhamento, nem nomeou as figuras, mas, na sonda de exclusão, foi capaz de reconhecer que o nome completamente novo não poderia se relacionar às figuras já apresentadas no livro, respondendo, portanto, por exclusão. Dessa forma, observa-se que a aprendizagem de novas palavras pode ser encarada com um contínuo de repertórios, que se inter-relacionam e apresentam dependência entre si. Investigar de que forma a exposição repetida das crianças às situações naturais promovem a experiência necessária para que esses repertórios se desenvolvam deve ser uma meta para todos os estudiosos do desenvolvimento da linguagem.

A presente pesquisa apresenta limitações que devem ser ponderadas na análise dos resultados: o número reduzido de participantes, a ausência de uma avaliação mais sistematizada do desenvolvimento das crianças e o pequeno número de sondas apresentadas em relação a todas as palavras investigadas são as mais importantes. Futuras pesquisas devem ampliar o número de crianças investigadas, assim como as faixas etárias estudadas, a fim de verificar se a experiência com a língua ou o tamanho do vocabulário das crianças têm influência na velocidade da aprendizagem, questão que pode ser levantada a partir da variabilidade dos resultados aqui encontrados. Além disso, é preciso considerar que podem ter ocorrido pequenas variações na forma da pesquisadora ler as histórias para as crianças, o que criaria efeitos não controlados, obscurecendo o efeito das dicas apresentadas. Sugere-se, por isso, que pesquisas futuras voltem a investigar o efeito de dicas sociais na situação de leitura compartilhada, assim como de outras variáveis contextuais, como o efeito da apresentação da função de um objeto desconhecido, definida no cenário da história; a influência da presença de outros indivíduos durante a leitura; o papel da quantidade de palavras desconhecidas presente na mesma história; as consequências da apresentação de outros tipos de dicas sociais, isoladas e simultaneamente, planejando condições experimentais e de leitura mais complexas em suas investigações. 


\section{Referências}

Ard, L. M., \& Beverly, B. L. (2004). Preschool word learning during joint book reading: Effect of adult questions and comments. Communication Disorders Quarterly, 26(1), 17-28. doi:10.1177/ 15257401040260010101

Biemiller, A., \& Boote, C. (2006). An effective method for building meaning vocabulary in primary grades. Journal of Educational Psychology, 98(1), 44-62. doi:10.1037/0022-0663.98.1.44

Blewitt, P., Rump, K. M., Shealy, S. E., \& Cook, S. A. (2009). Shared book reading: When and how questions affect young children's word learning. Journal of Educational Psychology, 101(2), 294-304. doi:10.1037/a0013844

Booth, A. E., McGregor, K. K., \& Rohlfing, K. J. (2008). Socio-pragmatics and attention: Contributions to gesturally guided word learning in toddlers. Language Learning and Development, 4(3), 179-202.doi:10.1080/15475440802143091

Brown, R., Waring, R., \& Donkaewbua, S. (2008). Incidental vocabulary acquisition from reading, reading-while-listening, and listening. Reading in a Foreign Language, 20, 136-163.

Carey, S., \& Bartllet, E. (1978). Acquiring a single new word. Papers and Reports on Child Language Development, 15, 19-29.

Carpenter, M., Nagell, K., \& Tomasello, M. (1998). Social cognition, joint attention, and communicative competence from 9 to 15 months of age. Monographs for the Society of Research in Child Development, 63(4), 1-143. doi:10.2307/1166214

Cozby, P. (2003). Métodos de pesquisa em ciências do comportamento. São Paulo, SP: Atlas.

Dixon, L. S. (1977). The nature of control by spoken words over visual stimulus selection. Journal of the Experimental Analysis of Behavior, 27, 433442. doi:10.1901/jeab.1977.27-433

Dunn, L. M., \& Dunn, L. M. (1997). Peabody picture vocabulary test - Revised. Circle Pines, MN: American Guidance Service.

Elley, W. B. (1989). Vocabulary acquisition from listening to stories. Reading Research Quarterly, 24(2), 174-187. doi:10.2307/747863

Evans, M. A., \& Saint-Aubin, J. (2005). What children are looking at during shared storybook reading: Evidence from eye movement monitoring. Psychological Science, 16, 913-920. doi:10.1111/j.1467-9280.2005.01636.x

Evans, M. A., \& Saint-Aubin, J. (2013). Vocabulary acquisition without adult explanations in repeated shared book reading: An eye movement study. Journal of Educational Psychology, 105, 596-608. doi:10.1037/a0032465

Farrant, B. M., \& Zubrick, S. R. (2013). Parentchild book reading across early childhood and child vocabulary in the early school years: Findings from the Longitudinal Study of Australian Children. First Language, 33, 280-293. doi:10.1177/0142723713487617

Flores, E. P., Pires, L. F., \& Souza, C. B. A. (2014). Dialogic reading of a novel for children: Effects on text comprehension. Paidéia (Ribeirão Preto), 24, 243-251. doi:10.1590/198243272458201412

Ganea, P. A., Allen, M. L., Butler, L., Carey, S., \& DeLoache, J. S. (2009). Toddlers' referential understanding of pictures. Journal of Experimental Child Psychology, 104, 283-295. doi:10.1016/j. jecp.2009.05.008

Greer, R. D., \& Du, L. (2015). Experience and the onset of the capability to learn names incidentally by exclusion. The Psychological Record, 65, 355-373. doi:10.1007/s40732-014-0111-2

Halberda, J. (2006). Is this a dax which I see before me? Use of the logical argument disjunctive syllogism supports word-learning in children and adults. Cognitive Psychology, 53, 310-344. doi:10.1016/j.cogpsych.2006.04.003

Heibeck, T. H., \& Markman, E. M. (1987). Word learning in children - An examination of fast mapping. Child Development, 58, 1021-1034. doi: $10.2307 / 1130543$

Horst, J. S. (2013). Context and repetition in word learning. Frontiers in Psychology, 4(149), 1-29. doi:10.3389/fpsyg.2013.00149

Horst, J., Parsons, K., \& Bryan, M. (2011). Get the story straight: Contextual repetition promotes word learning from storybooks. Frontiers in Developmental Psychology, 2(17), 1-11. doi:10.3389/fpsyg.2011.00017

Justice, L. M. (2002). Word exposure conditions and preschoolers' novel word learning during shared storybook reading. Reading Psychology, 23, 87-106. 
Justice, L. M., Skibbe, L. E., Canning, A., \& Lankford, C. (2005). Preschoolers, print, and storybooks: An observational study using eye-gaze analysis. Journal of Research in Reading, 28, 229-243. doi:10.1111/j.1467-9817.2005.00267.x

Kucirkova, N., Messer, D., \& Sheehy, K. (2014). Reading personalized books with preschool children enhances their word acquisition. First Language, 34, 227-243. doi:10.1177/0142723714534221

McIlvane, W. J., \& Stoddard, L. T. (1981). Acquisition of matching- to- sample performances in severe mental retardation: Learning by exclusion. Journal of Mental Deficiency Research, $25,33-48$.

Mintz, T. H., \& Gleitman, L. (2002). Adjectives really do modify nouns: The incremental and restricted nature of early adjective acquisition. Cognition, 84(3), 267-293.

Mol, S. E., Bus, A. G., de Jong, M. T., \& Smeets, D. J. (2008). Added value of dialogic parent-child book readings: A meta-analysis. Early Education and Development, 19, 7-26. doi:10.1080/10409280701838603

Oshiro, C. K. B., de Souza, D. G., \& Costa, A. R. A. (2006). Responder por exclusão a partir de uma linha de base de discriminações condicionais visuais. Revista Brasileira de Análise do Comportamento, 2, 251-276. doi:10.18542/rebac. v2i2.816

Robbins, C., \& Ehri, L. C. (1994). Reading storybooks to kindergartners helps them learn new vocabulary words. Journal of Educational Psychology, 86, 54-64. doi:10.1037/0022-0663.86.1.54

Rowe, M. L. (2000). Pointing and talk by lowincome mothers and their 14-month-old children. First Language, 20, 305-330. doi:10.1177/014272370002006005

Skinner, B. F. (1957). Verbal behavior. New York: Appleton-Century-Crofts.

Stemmer, N. (1996). Listener behavior and ostensive learning. Journal of the Experimental Analysis of Behavior, 65, 247-249. doi:10.1901/ jeab.1996.65-247

Thoermer, C., \& Sodian, B. (2001). Preverbal infants' understanding of referential gestures. First Language, 21(63), 245-264. doi:10.1177/014272370102106303
Tomasello, M., Carpenter, M., \& Liszkowski, U. (2007). A new look at infant pointing. Child Development, 78(3), 705-722. doi:10.1111/j.14678624.2007.01025.x

Vichi, C., Nascimento, G. S., \& Souza, C. B. A. (2012). Aprendizagem ostensiva, comportamento de ouvinte e transferência de função por pareamento de estímulos. Revista Brasileira de Terapia Comportamental e Cognitiva, 14, 16-30.

Wilkinson, K. M., de Souza, D. G., \& McIlvane, W. J. (2000). Origens da exclusão. Temas em Psicologia, 8, 195-203.

Wilkinson, K. M., Dube, W. V., \& Mcllvane, W. J. (1998). Fast mapping and exclusion (emergent matching) in developmental language, behavior analysis, and animal cognition research. The Psychological Record, 48, 407-422.

Wilkinson, K. S., \& Houston-Price, C. (2013). Once upon a time, there was a pulchritudinous princess...: The role of word definitions and multiple story contexts in children's learning of difficult vocabulary. Applied Psycholinguistics, 34(3), 591-613. doi:10.1017/S0142716411000889

Yu, C., \& Ballard, D. H. (2007). A unified model of early word learning: Integrating statistical and social cues. Neurocomputing, 70, 2149-2165. doi:10.1016/j.neucom.2006.01.034

Yu, C., \& Smith, L. B. (2012). Embodied attention and word learning by toddlers. Cognition, 125, 244-262. doi:10.1016/j.cognition.2012.06.016

Zucker, T. A., Cabell, S. Q., Justice, L. M., Pentimonti, J. M., \& Kaderavek, J. N. (2013). The role of frequent, interactive prekindergarten shared reading in the longitudinal development of language and literacy skills. Developmental Psychology, 49, 1425-1439. doi:10.1037/a0030347 